\title{
PROGRAMA MAIS MÉDICOS EM PERNAMBUCO E AS INTERNAÇÕES POR CONDIÇÕES SENSÍVEIS A ATENÇÃO PRIMÁRIA NOS MUNICÍPIOS DO G100.
}

\author{
E. S. Q. ESTIMA ${ }^{1}$, A. F. B. BEZERRA ${ }^{1,2}$ \\ ${ }^{1}$ Universidade Federal de Pernambuco, Centro de Ciências Sociais Aplicadas, Departamento \\ de Economia, Programa de Pós Graduação em Gestão e Economia da Saúde \\ ${ }^{2}$ Universidade Federal de Pernambuco, Centro de Ciências da Saúde, Departamento de \\ Medicina Social \\ E-mail para contato: manuqueirozz@hotmail.com
}

RESUMO- A Estratégia Saúde da Familia é a principal porta de entrada ao sistema de saúde brasileiro e é composto por uma equipe multiprofissional como: médico, enfermeiro, cirurgião dentista e agentes comunitários de saúde. O elevado aporte de médicos propiciado pelo Programa Mais Médicos tem o potencial de contribuir para a ampliação do acesso da população aos serviços de saúde e promover a melhoria da qualidade da assistência. Portanto, o fortalecimento da APS a partir do programa é, sobretudo, esperado nos municipios que integram o g100, caracterizados por grande porte populacional, baixa receita per capita e alto risco de vulnerabilidade socioeconômica. No entanto, como se trata de um programa recente, pouco se sabe sobre a efetividade do PMM nesse nivel de atenção. O objetivo deste trabalho foi verificar a produção científica sobre o programa relacionado a efetividade deste na atenção primária e sua contribuição para a redução das internações em Pernambuco segundo as internações por CSAP. A metodologia utilizada foi a revisão de literatura que aconteceu nas bases Google Scholar, ScientificElectronic Library Online, Literatura LatinoAmericana e do Caribe em Ciências da Saúde, Literatura Internacional em Ciências da Saúde. Espera-se a partir desta revisão desenvolver um estudo de avaliação que possa gerar informações úteis ao planejamento e a gestão da APS.

Palavras-chave: Políticas públicas, saúde, gestão.

ABSTRACT- The Family Health Strategy is the main gateway to the Brazilian health system and is composed of a multiprofessional team such as: doctor, nurse, dentist surgeon and community health agents. The high number of physicians provided by the Mais Médicos Program has the potential to contribute to the expansion of the population's access to health services and promote the improvement of the quality of care. Therefore, the strengthening of PHC from the program is mainly expected in the municipalities that integrate the g100, characterized by large population size, low per capita income and high risk of socioeconomic vulnerability. However, since this is a recent program, little is known about the effectiveness of PMM in this level of attention. The objective of this study was to verify the scientific production on the program related to its effectiveness in primary care and its contribution to the reduction of hospitalizations in Pernambuco according to hospitalizations for CSAP. The methodology used was the literature review that happened in the bases of Google Scholar, Scientific Electronic 
Library Online, Latin American and Caribbean Literature in Health Sciences, International Literature in Health Sciences. It is expected from this review to develop an evaluation study that can generate useful information for planning and managing PHC.

Keywords: Public policies, health, management.

\section{INTRODUÇÃO}

A Estratégia Saúde da Família (ESF) é o principal modelo de organização da APS no Brasil, e deve ser a porta de entrada preferencial do Sistema Único de Saúde (SUS) que garanta acesso universal, integral e equânime a comunidade (BRASIL, 2015).

No entanto, existem alguns desafios que dificultam a expansão da ESF e a resolutividade na atenção, como: a fragmentação do indivíduo, a baixa participação da comunidade, a dificuldade no acesso, infraestrutura inadequada, número insuficiente de médicos, regulação do sistema, falta de ações programáticas, e de delineamento dos casos (ELLERY et al, 2013).

O Governo Federal vem desenvolvendo ações e iniciativas que têm como objetivo facilitar e melhorar o acesso dos usuários do SUS à saúde de modo integral, tanto no âmbito gerencial do processo de trabalho, como na articulação de redes assistenciais integradas. Pode-se então destacar como parte integrante dessas ações, a criação do Programa Mais Médicos (PMM), em julho de 2013, por meio de Medida Provisória, que foi convertida na Lei $\mathrm{n}^{\circ} 12.871$ de 22 de outubro do referido ano (BRASIL, 2015).

O Mais Médicos foi organizado em 3 eixos: investimento e melhoria da estrutura da rede de saúde, ampliação e reformas educacionais dos cursos de graduação em medicina e a provisão de médicos em áreas vulneráveis no país (OLIVEIRA et al, 2015). Dentre os estados brasileiros que receberam médicos pelo PMM, o Nordeste foi o estado que obteve maior número de profissionais nos cinco primeiros ciclos da adesão (GONÇALVES et al.; 2016).

Segundo a Frente Nacional dos Prefeitos, 2015, no Brasil, 17\% dos municípios registraram a distribuição da renda anual por habitante inferior a $\mathrm{R} \$ 1.000$. Bahia $\mathrm{e}$ Pernambuco estão entre os estados que mais concentram municípios com baixa renda per capita, 39\% e 52\%, respectivamente. Dos municípios com renda inferior a $\mathrm{R} \$ 1.000$ por ano, 100 deles possuem população superior a 80 mil habitantes, classificados como pertencentes ao g100, sendo 40 da Região Nordeste, destes, 36 receberam médicos pelo PMM.

Assim sendo, estudos têm sido realizados a fim de avaliar a efetividade e os efeitos deste programa na APS. Um dos instrumentos comumente utilizado para avaliar a efetividade da APS em diferentes contextos é a observação das internações por Condições Sensíveis à Atenção Primária (CSAP), visto que altas taxas de internações por CSAP, podem indicar sérios problemas no acesso ao sistema de saúde ou em seu desempenho (ALFRADIQUE, et al 2009).

Logo, o objetivo deste trabalho foi verificar a produção científica atual sobre o programa relacionando a efetividade deste e sua contribuição na redução das internações em Pernambuco e assim observar a necessidade de trabalhos que analisem a ação do PMM nos municípios que integram o g100, segundo as internações por CSAP.

\section{MATERIAIS E MÉTODOS}

Trata-se de um estudo descritivo do tipo revisão de literatura sobre o Programa Mais Médicos e a ocorrência de internações por CSAP em municípios do g100 e que fazem 
parte do estado de Pernambuco. Segundo dados do Censo 2010 (IBGE, 2010), a população pernambucana naquele ano foi de 8.796 .448 habitantes, o que equivale a uma densidade demográfica de 89,63 habitantes $/ \mathrm{km}^{2}$.

Os 16 municípios do estado de Pernambuco inclusos na lista do g100 2017 como mostra a Tabela 1.

Tabela 1 - Municípios pertencentes ao g100 2017 e receita corrente per capta média.

\begin{tabular}{|c|c|c|c|}
\hline $\mathbf{U F}$ & MUNICÍPIOS & POPULAÇÃO 2015 & $\begin{array}{c}\text { RECEITA CORRENTE } \\
\text { PER CAPITA MÉDIA } \\
\text { 2013-2015 }\end{array}$ \\
\hline \multirow[t]{16}{*}{$\mathrm{PE}$} & Abreu e Lima & 98.602 & $\mathrm{R} \$ 1.293$ \\
\hline & Araripina & 82.800 & $\mathrm{R} \$ 1.592$ \\
\hline & Camaragibe & 154.054 & $\mathrm{R} \$ 1.386$ \\
\hline & Carpina & 81.054 & $\mathrm{R} \$ 1.313$ \\
\hline & Caruaru & 347.088 & $\mathrm{R} \$ 1.505$ \\
\hline & Garanhuns & 136.949 & $\mathrm{R} \$ 1.633$ \\
\hline & Gravatá & 81.893 & $\mathrm{R} \$ 1.446$ \\
\hline & Igarassu & 112.463 & $\mathrm{R} \$ 1.464$ \\
\hline & $\begin{array}{c}\text { Jaboatão dos } \\
\text { Guararapes }\end{array}$ & 686.122 & $\mathrm{R} \$ 1.474$ \\
\hline & Olinda & 389.494 & $\mathrm{R} \$ 1.345$ \\
\hline & Paulista & 322.730 & $\mathrm{R} \$ 1.088$ \\
\hline & Petrolina & 331.951 & $\mathrm{R} \$ 1.517$ \\
\hline & $\begin{array}{l}\text { Sta. Cruz do } \\
\text { Capibaribe }\end{array}$ & 101.485 & $\mathrm{R} \$ 1.236$ \\
\hline & São Lourenço da Mata & 110.264 & $\mathrm{R} \$ 1.314$ \\
\hline & Serra Talhada & 84.352 & $\mathrm{R} \$ 1.493$ \\
\hline & Vitória & 135.805 & $\mathrm{R} \$ 1.621$ \\
\hline
\end{tabular}

Fonte: Frente Nacional de Prefeitos, 2017

Para desenvolvimento do estudo foram analisados artigos científicos encontrados na base de dados: Google Scholar, Scientific Electronic Library On-line (SciELO), Literatura Latino-Americana e do Caribe em Ciências da Saúde (LILACS), Literatura Internacional em Ciências da Saúde (MEDLINE) que correspondam ao período de 2013 a 2017 e utilizados dados secundários oriundos do Projeto Mais Médicos para o Brasil, do sistema de informação do Departamento de Atenção Básica, DATASUS, e consulta a lista de CSAP.

O período escolhido é devido ao ano que o Programa Mais Médicos foi implantado no Brasil, julho de 2013. Os critérios de inclusão utilizados na busca foram: teses, dissertações, documentos oficiais, capítulos de livros, notícias editoriais, artigos na íntegra on-line; em línguas portuguesa e inglesa, que utilizaram o termo "Programa Mais Médicos", indexado nas bases de dados citadas, publicados no período de 2013 a 2017. Os critérios de exclusão foram os textos não científicos. A pesquisa foi realizada a partir de títulos, resumos, palavras-chave e texto completo. 


\section{RESULTADOS E DISCUSSÃO}

Como resultado da busca, utilizando as palavras "Programa mais Médicos", nas bases de dados selecionadas foram encontrados: LILACS, 67; MEDLINE, 1722 artigos; SciELO, 70 artigos; Google Scholar, 977 artigos.

Segundo Ministério da Saúde (BRASIL, 2015, p. 15):

"A Atenção Básica é a porta de entrada preferencial do Sistema Único de Saúde (SUS), que deve ordenar o acesso com equidade aos demais serviços da rede de saúde, estar presente em todos os municípios e próxima de todas as comunidades, garantindo acesso universal, com qualidade e em tempo oportuno".

No Brasil, a Estratégia Saúde da Família é o principal modelo de organização da APS. Surgiu em 1994, como o objetivo de melhorar a situação das famílias brasileiras, propondo a princípio a reorientação no modelo de atenção por meio de equipes multiprofissionais nas Unidades Básicas de Saúde (UBS), ou Programa Saúde da Família (PSF), como eram chamadas no início (SOUZA et al, 2012).

Segundo a Política Nacional da Atenção Básica (2012) a atenção primária deve ser desenvolvida com o mais alto grau de descentralização, para estar próxima da vida das pessoas. É norteada pelos princípios da universalidade, da acessibilidade, do vínculo da continuidade do cuidado, da integralidade da atenção, da universalização, da humanização, da equidade e da participação social.

Este vínculo de comunicação entre a população e a rede é permitida por meio do contato mantido entre a população e a equipe do Saúde da Família - EqSF, a qual é composta por médico, enfermeiro, técnico de enfermagem e agentes comunitários de saúde (ACS), e quando ampliada conta ainda com um dentista, um auxiliar de consultório dentário e um técnico em higiene dental (BRASIL, 2012).

Estes profissionais devem atuar de maneira interdisciplinar, responsabilizando-se pelo território, focando a intervenção sobre as necessidades específicas das famílias e atender aos problemas da comunidade. Porém, existem alguns desafios para a expansão da ESF, como: a fragmentação do indivíduo, mantidas pelo isolamento e pelas relações de poder entre os profissionais e com usuários, a baixa participação da comunidade, dificuldade no acesso, infraestrutura inadequada, regulação do sistema, falta de ações programáticas, e de delineamento dos casos, o que inviabiliza a produção do trabalho em equipe e a uma atenção à saúde integral e resolutiva (ELLERY, et al 2013).

O Ministério da Saúde (2015) indica que a expansão da Estratégia de Saúde da Família apresentou, nos anos de 2006 a 2013, um aumento de cobertura de apenas 1,5\% da população a cada ano, segundo informações do Departamento de Informática do SUS (DATASUS), sendo a dificuldade dos gestores municipais em atrair e fixar médicos nas equipes de Saúde da Família (eSF) um dos fatores que dificultaram essa expansão.

Em função da dificuldade de fixação de médicos em várias regiões do país, o Ministério da Saúde juntamente ao Ministério da educação implementou ações que buscam intervir nesses desafios enfrentados pela ESF (BRASIL, 2015).

Desde 1979 várias estratégias eram desenvolvidas para o provimento de médicos nos serviços de saúde, como: o Programa de Interiorização das Ações de Saúde e Saneamento - PIASS; o Programa de Interiorização do Sistema Único de Saúde - PISUS e o Programa de Interiorização do Trabalho em Saúde e implantação de internatos rurais nas graduações da área de saúde. (NOGUEIRA, 2016)

Em 2011, foi desenvolvido o Programa de Valorização do Profissional na Atenção Básica (PROVAB), ofertando a possibilidade de trabalho supervisionado em regiões de 
extrema pobreza e periferias, com incentivo aos participantes de acréscimo na pontuação no processo seletivo da residência médica, ainda assim não conseguiu atender toda a necessidade dos municípios, dando origem a campanha Cadê o Médico da Frente Nacional de Prefeitos 2013 (BRASIL, 2015).

Como resposta, surge o Programa Mais Médicos para o Brasil, criado em julho de 2013, por meio de medida Provisória, que foi convertida em Lei em outubro do mesmo ano, após muitos debates que refletiam as necessidades da sociedade e também discussões no Congresso Nacional (BRASIL, 2015).

De acordo com o Ministério da Saúde (BRASIL, 2015) embora não exista parâmetro que estabeleça uma proporção ideal de médico por habitante reconhecido e validado internacionalmente, a referência usada para o Programa Mais Médicos foi a meta de 2,7 médicos por 1.000 habitantes de acordo com outros países, como o Reino Unido que apresenta, seguido ao Brasil, o maior sistema público de saúde de caráter universal orientado pela atenção básica. Verificou-se então que o número de médicos por habitante se encontrava abaixo de outros países e da média dos países da Organização para a Cooperação Econômica e Desenvolvimento que é de 3,2.

A distribuição de médicos nos estados brasileiros configurava anteriormente ao lançamento do Programa uma grande desproporção, segundo os dados do IBGE 2012 e dados oriundos do Conselho Federal Médico (CFM), pois apenas em cindo destes tinham mais de 1,83 médico por 1.000 habitantes (BRASIL, 2015).

O PMM, destinado a provisão de profissionais para atuação nos municípios com maior necessidade e dificuldade de fixação de profissionais e maior vulnerabilidade. As regiões foram classificadas por prioridade como: áreas com percentual elevado de população em extrema pobreza; baixo índice de desenvolvimento humano ou regiões muito pobres; semiárido e região amazônica; áreas com população indígena e quilombola (BRASIL,2013).

Os médicos que estariam aptos a participar seriam aqueles que tivessem concluído a graduação no Brasil ou no exterior, estrangeiros graduados no Brasil ou no exterior, desde que vindos de países cujo número de médicos per capita fosse acima de 1,8 por mil, que corresponde ao número de médicos per capita no Brasil antes do PMMB (BRASIL, 2013).

O Ministério da Saúde divulgou após aproximadamente dois anos de lançamento do programa que 18.240 médicos participavam do projeto em 4.058 municípios $(72,9 \%$ dos municípios do Brasil), além de 34 distritos indígenas, beneficiando cerca de 63 milhões de pessoas. Dentre as regiões brasileiros destaca-se o Nordeste com maior adesão de médicos e início das atividades profissionais representando 33,4\%, seguido pelo Sudeste com proporção de $30 \%$, Sul $(16,6 \%)$, Norte $(13,1 \%)$ e Centro-Oeste $(6,4 \%)$ (BRASIL, 2016).

Embora tenha ocorrido uma expansão considerável no quantitativo dos profissionais médicos nos últimos anos, o país ainda apresenta disparidades quanto a sua distribuição frente ao número populacional e quanto a APS a região Nordeste apresenta a maior necessidade, onde $49,3 \%$ da população reside em área com escassez deste profissional (NOGUEIRA et al 2016).

O Ministério da Saúde (BRASIL, 2015) referiu que os quatro piores indicadores quanto a distribuição de médicos se encontravam no Nordeste na relação médico por 1000 habitantes, como Bahia (1,09), Ceará (1,05 médico/1000 hab.), Piauí (0,92) e Maranhão $(0,58)$. O estado de Pernambuco apresentou o maior indicador de distribuição no NE $(1,39)$.

Pinto et al (2014) afirma que: 
Quanto menor, mais pobre, de difícil acesso e precárias as condições social, econômica e sanitária da região e do município, maior é a necessidade de médicos, maior é a escassez desse profissional, mais inflacionado é o mercado de trabalho médico da região e menor é a capacidade do município de atrair e fixar os profissionais (p.109).

Considerando os critérios do Programa, a lotação dos médicos nas ESF deve ser prioritariamente em áreas vulneráveis dos municípios brasileiros, como áreas referentes aos $40 \%$ dos setores censitários com maiores percentuais de população em extrema pobreza ou municípios com $20 \%$ ou mais nesta situação de vulnerabilidade, e municípios que contenham áreas pertencentes a Distritos Sanitários Especiais Indígenas (DSEI) também é considerado prioritário, estratifica-se ainda os municípios seguindo a classificação de capitais, regiões metropolitanas e g100 ( RIBAS, 2016)

Abaid (2014) defende que a forma de orientar a gestão e as políticas de saúde é avaliando e monitorando a efetividade da APS, propondo como indicador avaliativo as hospitalizações por CSAP.

Embora os estudos com as ICSAP tenham contribuído para revelar as evidências de efetividade da APS, a maior parte deles são voltados a municípios de grande porte (acima de 100 mil habitantes) (GONÇALVES et al, 2016).

Em Pernambuco, Mendonça e Albuquerque (2014) observaram que a distribuição dos serviços de atenção à saúde ainda é heterogênea, concentrada na região metropolitana do Recife, o que dificulta o acesso da população interiorana aos diferentes níveis de atenção do Sistema Único de Saúde (SUS).

Nesse contexto se insere os municípios do estado de Pernambuco que compõe o g100 como local de interesse do estudo, pois se trata de cidades com população superior a 80 mil habitantes que não são beneficiadas pela distribuição do Fundo de Participação dos Municípios (FPM), devido ao porte populacional dos municípios, tendo então uma formação econômica fragilizada e crescimento demográfico significativo (FNP, 2015).

De acordo com a FNP (2017), o índice g100 é composto dos seguintes critérios com suas ponderações:

- Índice da Receita Corrente Per Capita: ponderado em 70\%.

-Índice de Pobreza: ponderado em 10\%.

•Índice da Independência da População em relação ao Sistema Único de Saúde (SUS): ponderado em 10\%.

- Índice da Educação: ponderado em 10\%

O PMM, tornou o g100 critério oficial para a alocação de médicos provenientes do programa, o que destaca sua importância e relevância, e é considerado como elemento fundamental na correção das distorções que programas federais podem ocasionar (FNP, 2015).

\section{CONCLUSÃO}

O PMM trata-se de um programa recente, que pouco se sabe sobre sua efetividade na APS. Desse modo, espera-se a partir desta revisão desenvolver um estudo de avaliação que possa gerar informações úteis ao planejamento e a gestão da APS sob a perspectiva avaliativa do desempenho do programa como um indicador de efetividade da Estratégia Saúde da Família na redução das internações por condições sensíveis a atenção primária de saúde frente ao cenário socioeconômico destes municípios. Assim como, despertar o olhar do público para desenvolvimento de outros trabalhos acerca do programa e sobre o que ainda se faz necessário para se ampliar o acesso e garantir o direito à saúde no país. 


\section{REFERÊNCIAS}

ABAID, R. A.; NEDEL, F. B.; ALCAYAGA, E. L. Condições sensíveis à atenção primária: confiabilidade diagnóstica em Santa Cruz do Sul, RS. Revista de Epidemiologia e Controle de Infecção, v. 4, n. 3, Brasília, 2014.

ALFRADIQUE M.E; BONOLO P.F; DOURADO I; LIMA-COSTA M.F; MACINKO J; MENDONÇA CS; OLIVEIRA V.B; SAMPAIO L.F.R; SIMONI C.D; TURCI M.A. Internações por condições sensíveis à atenção primária: a construção da lista brasileira como ferramenta para medir o desempenho do sistema de saúde (Projeto ICSAP - Brasil). Caderno de Saúde Publica, 2009.

BRASIL. Lei n. 12.871, de 22 de outubro de 2013. Institui o Programa Mais Médicos, altera a lei 8.745, de 9 de dezembro de 1993, e $n^{\circ} 6.932$, de 7 de julho de 1981, e da outra providência. Disponível em: <http://www.planalto.gov.br/ ccivil_03/_ato20112014/2013/Lei/L12871.htm>. Acesso em: 15 de novembro de 2016.

BRASIL. Ministério da Saúde. Secretaria de Gestão do Trabalho e da Educação na Saúde. Edital $n^{\circ}$ 50, de 16 de agosto de 2013. Adesão dos médicos ao Projeto Mais Médicos para o Brasil. Diário Oficial da União. 19 agosto 2013. Disponível em: http://portalsaude.saude.gov.br/index.php/cidadao/acoes-e-programas/mais-medicos/ mais-sobre-mais-medicos/5955-documentos. Acesso em 16 de março de 2017.

BRASIL. Ministério da Saúde. Secretaria de Atenção à Saúde. Departamento de Atenção Básica. Política Nacional de Atenção Básica. Brasília, 2012.

BRASIL. Ministério da Saúde. Secretaria de Gestão do Trabalho e da Educação na Saúde. Programa mais médicos - dois anos: mais saúde para os brasileiros. Brasília, 2015.

ELLERY, A. E. L; PONTES, R. J. S; LOIOLA, F. A. Campo comum de atuação dos profissionais da Estratégia Saúde da Família no Brasil: um cenário em construção. Physis, Rio de Janeiro,v. 23, n. 2, p.415-437, Junho 2013. Disponível em: http://www.scielo.br/scielo.php?script=sci arttext\&pid=S01033312013000200006\&lng $=$ en\&nrm=iso.

Frente Nacional dos Prefeitos (FNP). G100 Municípios Populosos com Baixa Receita Per Capita e Alta Vulnerabilidade Social. Florianópolis: FNP; 2015.

Frente Nacional dos Prefeitos (FNP). G100 Municípios Populosos com Baixa Receita Per Capita e Alta Vulnerabilidade Social. Florianópolis: FNP; 2017.

GONÇALVES, F. R; SOUZA, I. M. C. S; TANAKA, O. Y; SILVA, K. B; SANTOS, L. X.; BEZERRA, A. F. B. Programa Mais Médicos no Nordeste: avaliação das internações por condições sensíveis à Atenção Primária à Saúde. Rev. Ciência \& Saúde Coletiva. 2016.

INSTITUTO BRASILEIRO DE GEOGRAFIA E ESTATÍSTICA (IBGE). Censo Brasileiro de 2010. Rio de Janeiro: IBGE, 2012.

NOGUEIRA, P. T. A; BEZERRA, A. F. B; LEITE, A. F. B; CARVALHO, I. M. S; GONÇALVES, R. F; BRITO-SILVA, K. S. Características da distribuição de 
profissionais do Programa Mais Médicos nos estados do Nordeste, Brasil. Ciência \& Saúde Coletiva, 2016.

OLIVEIRA F.P, VANNI T, PINTO H.A, SANTOS J.T.R, FIGUEIREDO A.M, ARAÚJO S.Q, MATOS M.F.M, CYRINO E.G. Mais Médicos: um programa brasileiro em uma perspectiva internacional. Interface, Botucatu, 2015.

PINTO, H. A; SALES, M. J. T; OLIVEIRA, F. P; BRIZOLARA, R; FIGUEIREDO, A. M.; SANTOS, J. T. O Programa Mais Médicos e o fortalecimento da Atenção Básica Mais Médicos. Divulgação em saúde para debate. Rio de Janeiro, n. 51, p. 105-120, out 2014.

RIBAS, A. N. Programa Mais Medicos: uma avaliação dos resultados iniciais referentes ao eixo do provimento emergencial a partir da Teoria da Avaliação de Programas. 2016.

SouzA, B. P. A; PAUlette, A. C. Projeto Mais Médicos para o Brasil em Pernambuco: uma abordagem inicial. Tempus Actas de Saúde Coletiva, v. 9, n. 4, p. 11-34, 2015. 